\title{
Erratum to: Acoustic radiation force due to incident plane-progressive waves on coated spheres immersed in ideal fluids
}

\section{F.G. Mitri ${ }^{\mathrm{a}}$}

College of Medicine, Mayo Clinic, Department of Physiology and Biomedical Engineering, Ultrasound Research Laboratory, 200 First Street SW, Rochester MN 55905, USA

\section{Received 3 May 2010}

Published online 24 June 2010 - (C) EDP Sciences, Società Italiana di Fisica, Springer-Verlag 2010

Eur. Phys. J. B 43, 379 (2005)

Concerning equations (13), (15) as well as the expressions for $\left\langle F_{t}\right\rangle$ in (19) and the radial $U_{r}^{(2,3)}$ and angular component $U_{\theta}^{(2,3)}$ of the displacement vector $\mathbf{U}$ inside the layered sphere in Appendix A in [1], an error of transcription occurred.

The functions $\Psi_{2}$ and $\Psi_{3}$ given by (13) and (15), respectively, should have been printed as,

$$
\begin{aligned}
\Psi_{2}(r, \theta)=\Phi_{0} \sum_{n=1}^{\infty} i^{n} & (2 n+1)\left(D_{n} j_{n}\left(k_{S, 2} r\right)\right. \\
& \left.+E_{n} n_{n}\left(k_{S, 2} r\right)\right) \frac{d}{d \theta}\left(P_{n}(\cos \theta)\right),
\end{aligned}
$$

and

$\Psi_{3}(r, \theta)=\Phi_{0} \sum_{n=1}^{\infty} i^{n}(2 n+1) G_{n} j_{n}\left(k_{S, 3} r\right) \frac{d}{d \theta}\left(P_{n}(\cos \theta)\right)$.

The expression for $\left\langle F_{t}\right\rangle$ in (19) should have been printed as

$$
\left\langle F_{t}\right\rangle=-\frac{\pi c^{2} \rho_{1}}{c_{1}^{2}}\left\langle\int_{0}^{\pi}\left(\frac{\partial \varphi_{1}}{\partial t}\right)_{r=c}^{2} \sin \theta \cos \theta d \theta\right\rangle .
$$

a Current address: Los Alamos National Laboratory, MPA11, Sensors \& Electrochemical Devices, Acoustics \& Sensors Technology Team, MS D429, Los Alamos, NM 87545, USA, e-mail: mitri@lanl.gov
Finally the expressions for $U_{r}^{(2,3)}$ and $U_{\theta}^{(2,3)}$ in Appendix A should have been printed as:

$$
U_{r}^{(2,3)}=\frac{\partial \Phi_{2,3}}{\partial r}+\frac{1}{r \sin \theta} \frac{\partial\left(\Psi_{2,3} \sin \theta\right)}{\partial \theta}
$$

and

$$
U_{\theta}^{(2,3)}=\frac{1}{r} \frac{\partial \Phi_{2,3}}{\partial \theta}-\frac{1}{r} \frac{\partial\left(r \Psi_{2,3}\right)}{\partial r},
$$

respectively. These changes bring the above-mentioned expressions in agreement with expressions given by various other authors. It is important to emphasize that the derivations leading to the determination of the scattering coefficients $A_{n}$ and their corresponding matrix elements in Appendix B, as well as the computational plots shown in [1] used the correct expressions for $\Psi_{2}, \Psi_{3},\left\langle F_{t}\right\rangle, U_{r}^{(2,3)}$ and $U_{\theta}^{(2,3)}$.

\section{References}

1. F.G. Mitri, Eur. Phys. J. B 43, 379 (2005) 